\title{
El uso de Twitter en el entorno del Periodismo Institucional 2.0: estrategias cross-media y diálogo informativo ${ }^{1}$
}

\section{Twitter and public agencies 2.0: cross-media strategies and informative dialogue}

Sonia González-Molina

Profesora (Área de Periodismo, Departamento de Ciencias de la Comunicación, Universitat Jaume I)

Fecha de recepción: 30 de marzo de 2013

Fecha de revisión: 22 de abril de 2013

Para citar este artículo: González-Molina, S. (2013): El uso de Twitter en el entorno del Periodismo Institucional 2.0: estrategias cross-media y diálogo informativo, Icono 14, volumen 11 (2), pp. 141-162. doi: 10.7195/ri14. v11i2.582 


\section{Resumen}

El Periodismo Institucional está redefiniendo sus canales de comunicación externos de la mano de Twitter, un servicio microblogging en auge. El artículo analiza el uso de esta herramienta en este campo, en un contexto marcado por la convergencia, que incentiva las estrategias cross-media y el diálogo con los usuarios. Se basa en la experiencia del gabinete de prensa del Servei Català de Trànsit (SCT), la principal fuente sobre movilidad en Catalunya. La metodología escogida para desarrollar el trabajo es la del estudio de caso. Concretamente, combina la entrevista con el análisis del contenido de los tuits difundidos por el organismo durante la segunda quincena del mes de junio de 2012. La principal conclusión apunta a que Twitter se utiliza básicamente como complemento a los contenidos distribuidos a través de la página web, sin apenas opciones al diálogo público ni con usuarios ni con periodistas.

\section{Palabras clave}

Twitter - Periodismo Institucional - Gabinetes de prensa - Cross-media - Convergencia - Diálogo

\section{Abstract}

Public agencies are reshaping its communication channels through Twitter, a microblogging service which is reaching its peak. This article analyses how the press offices of institutional organizations are using this tool in the general context of the convergence, which encourages the cross-media strategies and the dialogue with the public. It is based on the case study of the Servei Català de Trànsit (SCT), the main official source about road information in Catalonia. It mixes an interview with the content analysis of the tweets distributed by the organism during the last two weeks of June 2012. The main conclusion of this work is that Twitter is basically an informative complement of the corporate website. There is no option to public dialogue neither with the public nor with the journalists.

ICONO14 | Año 2013 Volumen 11 N² 2 | ISSN: 1697-8293 | DOI: ri14.v1 1i2.582 
El uso de Twitter en el entorno del Periodismo Institucional 2.0| 143

\section{Key Words}

Twitter - Public agencies - Press offices - Cross-media - Convergence - Dialogue

\section{Introducción}

La tecnología digital irrumpió en los gabinetes de prensa y comunicación a mediados de los años noventa del siglo pasado. Internet sedujo principalmente por su capacidad para almacenar un gran volumen de material, en múltiples formatos, y distribuirlo a gran velocidad al mismo tiempo que ofrecía a los organismos la posibilidad de dialogar directamente con sus públicos (Marken, 1995). La red se implantó definitivamente en el entorno laboral de estas oficinas al mismo ritmo al que lo hacía en los medios de comunicación, con unos periodistas que incorporaban con naturalidad a sus rutinas las nuevas herramientas tecnológicas a su alcance (Garrison, 2003).

La última innovación en irrumpir con fuerza en el ámbito laboral de las oficinas de prensa ha sido los medios sociales, con probada capacidad para modificar los mecanismos para producir y distribuir contenidos informativos entre los periodistas en particular y los ciudadanos, en última instancia. Por su naturaleza dialógica, se encuadran dentro de la denominada web 2.0, que se caracteriza por ofrecer participación y colaboración de los usuarios en la creación de contenido, frente a la anterior realidad, en la que sólo podían ejercer de observadores pasivos. Del conjunto de aplicaciones vinculadas con esta filosofía, este artículo se centra en Twitter, por su creciente protagonismo en aquellas actividades informativas dentro del entorno más genérico del Periodismo Institucional, entendido como aquel que se ocupa de los procesos informativos que se dan en el seno de las organizaciones, sean éstas públicas o privadas.

Desde el punto de vista de instituciones y empresas, los medios sociales se perfilan como una de las áreas con mayor proyección en las estrategias comunicativas. El último informe European Communication Monitor (Zerfass y otros: 2012), un estudio que desde 2007 analiza el estado de la comunicación corporativa en Europa a través de una encuesta masiva a profesionales de este sector, señala que tres cuartas partes de ellos identifican estas herramientas como relevantes en su trabajo. Más concretamente, el 75\% habla de las redes sociales en general y el 56\%

DOI: ri14.v1 1i2.582 | ISSN: 1697-8293 | Año 2013 Volumen 11 N² 2 | ICONO14 
de las plataformas de microblogging tipo Twitter. De hecho, los anteriores sondeos ya anunciaban el progresivo protagonismo de estas innovaciones (Moreno y otros, 2009, 2010; Verhoeven, 2012).

Con todo, el desfase respecto al uso de estas herramientas que revela este sondeo, ya que sólo una tercera parte de los encuestados asegura usar Twitter en su trabajo, aventura futuro recorrido para esta innovación. Se la ve como importante, aunque no ha alcanzado aún su culminación a la espera de encontrar un espacio propio dentro del conjunto de canales que los organismos emplean para su comunicación externa. Este entorno de reconfiguración desencadenado por la aparición de nuevas herramientas tecnológicas aconseja pues abordar cuáles son los usos de esta plataforma y las estrategias informativas que allí se siguen. Por ello, el objetivo de esta propuesta es el de explicar de qué manera utilizan Twitter los gabinetes de prensa de organismos públicos. Se enmarca en el denominado Periodismo Institucional, al que definiremos como aquella especialización que se ocupa de los procesos y prácticas informativas que se desarrollan en el seno de instituciones, empresas y asociaciones de diverso tipo, públicas o privadas. Como otros ámbitos de la comunicación, el Periodismo Institucional también se ve afectado por los procesos de convergencia y por el cambio de paradigma que marca el paso de una audiencia pasiva a otra activa y con capacidad de participar en el proceso informativo.

\section{Material y métodos}

Como acabamos de explicar, esta investigación se inscribe en el conjunto de propuestas que, desde la convergencia, han examinado la transformación del periodismo en sus vertientes empresarial, profesional, de contenidos y en relación con la audiencia. El punto de partida, en este caso, es la irrupción de los medios sociales en el ámbito de la comunicación, entendidos como una de las últimas innovaciones capaces de transformar nuevamente los mecanismos de producción y distribución informativas así como las competencias y habilidades de los periodistas y la relación con los usuarios.

Este es el esquema que habitualmente se ha utilizado para abordar la irrupción de la tecnología digital en los medios, siendo más escasos por no decir extraordinarios los ejemplos vinculados al Periodismo Institucional. No obstante, cada vez más gabinetes de prensa y comunicación incorporan las utilidades de la web 2.0 como

ICONO14 | Año 2013 Volumen 11 N² | ISSN: 1697-8293 | DOI: ri14.v1 1i2.582 
mecanismo de comunicación externa. En este contexto, Twitter se combina con otras herramientas, como la web corporativa o el correo electrónico, a las que complementa en estas funciones y con las que teje complicidades identificables bajo el paraguas de la noción de cross-media. $\mathrm{Y}$, de momento, estas estrategias apenas se han examinado en el trabajo informativo de las oficinas de prensa.

La adaptación que a esta nueva realidad está llevando a cabo el Periodismo Institucional es el motivo fundamental que justifica la pertinencia de este trabajo de investigación. Máxime, cuando el uso de Twitter apenas ha sido abordado en este ámbito de estudio desde esta perspectiva. Por ahora, se ha examinado su uso en funciones comunicativas de manera global, es decir, en el contexto de la relación de los organismos con los usuarios en general. Sin embargo, son prácticamente inéditas las aproximaciones al uso que hacen de esta plataforma los gabinetes de prensa institucionales, en pleno proceso de redefinición de sus canales de comunicación externa como consecuencia de la convergencia. He aquí el segundo motivo que explica esta propuesta.

Por tanto, el objetivo principal de este texto es explicar de qué manera utilizan Twitter los gabinetes de prensa institucionales, qué contenidos distribuyen a través de esta plataforma y por qué lo hacen así. Se hará a partir de la experiencia de la principal fuente de información oficial sobre tráfico y seguridad vial existente en Catalunya, el Servei Català de Trànsit (SCT). Su carácter pionero en la incorporación de las nuevas tecnologías en la difusión de información ${ }^{2}$ así como su centralidad en el mapa de fuentes que los periodistas catalanes que se ocupan de esta especialidad informativa así lo aconseja. Y permite aventurar una posible extrapolación a otros departamentos del mismo tipo.

La propuesta plantea dos hipótesis de investigación, relacionadas ambas con los aspectos planteados en el apartado teórico de este artículo: la primera, con las estrategias cross-media y la convergencia, y la segunda, con la comunicación dialógica. Se trata de las siguientes:

1. Las estrategias de distribución de contenidos entre los diferentes canales externos de comunicación de los gabinetes se basan en la reutilización de contenidos ya disponibles en la web, sin apenas adaptaciones.

2. A pesar de ser una herramienta eminentemente dialógica, los gabinetes implementan estrategias comunicativas que evitan la conversación pública con sus seguidores, entre los que se encuentran los periodistas.

DOI: ri14.v11i2.582 | ISSN: 1697-8293 | Año 2013 Volumen 11 N² | ICONO14 
La metodología utilizada se basa en el estudio de casos, una técnica de naturaleza cualitativa que permite combinar el uso de varias fuentes para la recopilación de datos (Castro Monge, 2010). Ello abre la puerta al planteamiento de generalizaciones analíticas para explicar y comprender los fenómenos estudiados (Yin, 1994). Merriam (1988) explica que el estudio de casos permite elaborar descripciones, ofrecer interpretaciones sobre lo que se investiga, explorar sus características y hacer una evaluación. Por ello, la elección de esta técnica se muestra como apropiada desde el punto de vista metodológico.

En este trabajo de investigación, se ha optado por una entrevista semi-estructurada con la responsable de prensa y comunicación del SCT a fin de conocer los objetivos que motivaron al organismo a incorporar Twitter a su trabajo y también las funciones que desarrolla esta plataforma en el interior del gabinete. Se completó con un análisis del contenido de los tuits enviados por este organismo durante dos semanas, del 18 de junio al 1 de julio de 2012. La muestra asciende a 763 tuits. Para su análisis, se elaboró una ficha específica que clasificó sus contenidos en función de tres variables: los temas tratados, los recursos utilizados (enlaces y material multimedia) y las marcas de relación con los seguidores (retuits y conversaciones públicas). Las categorías en que se dividieron los temas se definieron a partir la entrevista con la responsable de prensa del organismo y son cinco: "servicio" (información sobre movilidad), "sucesos" (víctimas y accidentes mortales), "servicio y sucesos" (combinación de las anteriores), "consejos" (mensajes preventivos) y "prensa" (actividades informativas del organismo).

El texto articula los resultados del trabajo de campo a partir de los mecanismos de producción y distribución informativa detectados, la gestión de los contenidos informativos y la relación con la audiencia desde el punto de vista de la estimulación del diálogo. Antes plantea el marco teórico, centrado en la convergencia, las estrategias cross-media y el uso de Twitter en el Periodismo Institucional.

\section{Marco teórico: convergencia y estrategias cross-}

\section{media}

Desde finales del siglo pasado, la convergencia ocupa un lugar central en los estudios sobre nuevas tecnologías y evolución del periodismo. Entendido como un

ICONO14 | Año 2013 Volumen 11 N² 2 | ISSN: 1697-8293 | DOI: ri14.v1 1i2.582 
proceso aún en desarrollo, este fenómeno hunde sus raíces en la digitalización y concentración informativa desarrollada desde los años noventa del siglo XX. Con frecuencia, la convergencia se utiliza para contextualizar los diversos cambios que, en los últimos años, han experimentado las empresas periodísticas, sus profesionales, los contenidos informativos y la relación con la audiencia (Infotendencias group, 2012). Deuze (2004) la define como la creciente colaboración y cooperación entre redacciones originalmente independientes, lo que acerca el ideal convergente a la integración plena de estas unidades de trabajo, según el modelo clásico de Dailey y otros (2005). La realidad es más flexible y enseña que cada medio aplica su propia receta en función de sus objetivos, intereses y maneras de trabajar.

Uno de los aspectos que se abordan bajo el paraguas de la convergencia tiene que ver con la posibilidad de que los medios, ya sea de manera individual o bien formando parte de un grupo de comunicación, distribuyan sus contenidos de manera coordinada a través de varios canales asociados entre sí. La denominada distribución multiplataforma se refiere al uso de múltiples soportes en lugar de uno solo para hacer llegar las noticias al público (Jenkins, 2008). Así, la difusión de contenidos se vuelve más eficiente y el medio, más omnipresente (Domingo y otros, 2007).

Prácticamente todas las investigaciones sobre convergencia que describen el trabajo y funcionamiento de las redacciones multimedia se detienen en el aspecto anteriormente citado. Aunque sólo sea de manera tangencial al presentarlo asociado a otros aspectos como la polivalencia (Wallace, 2013) o los modelos de negocio y nuevos atributos de la información digital (Casero-Ripollés, 2010, 2012). Como ejemplos a nivel nacional, podemos citar las investigaciones de García Avilés y otros (2004, 2009), Larrondo y otros (2012), Franquet y Villa Montoya (2012a) o Micó y otros (2013). A escala internacional, tenemos las aportaciones de Cottle y Ashton (1999), Marjoribanks (2003), Huang y otros (2004, 2006), Singer (2004), Boczkoswi (2004), Dupagne y Garrison (2006), y Erdal (2008), éste último, plenamente centrado en la noción de cross-media.

El anterior concepto, el de cross-media ${ }^{3}$, emerge ligado al de distribución multiplataforma y, por extensión, al de la propia convergencia. Ibrus y Scolari (2012) lo definen como aquella propiedad intelectual, servicio, historia o experiencia distribuida a través de múltiples plataformas mediáticas usando diversos formatos para ello. Por su parte, Erdal (2011) diferencia cross-media de multiplataforma cuando 
explica que, para que un contenido sea considerado cross-media, es necesario que aquéllas funcionen de forma integrada de manera que se necesite el concurso de todas las implicadas para un consumo informativo sin alteraciones en la producción final.

Para Ibrus y Scolari (2012), las prácticas cross-media representan un esfuerzo clave para los medios. Las sitúan dentro de las estrategias de diversificación de contenidos que Chan-Olmsted y Chang $(2003,2006)$ identificaron en el funcionamiento de los conglomerados multimedia en el contexto más amplio de la convergencia. Esto es, la adaptación de las piezas a las plataformas de distribución para reforzar su presencia pública a través de la promoción cruzada entre ellas y aumentar así el margen de beneficio.

En cualquier caso, las estrategias cross-media demandan un cierto esfuerzo a favor de la coordinación y cooperación interna del grupo mediático, y una mentalidad más abierta por parte de los periodistas, obligados a trabajar en un entorno en el que las tradicionales divisiones entre los medios se difuminan (García Avilés, 2012). Desde el punto de vista de los contenidos, implican prever cómo se distribuyen las piezas informativas a través de las diversas plataformas y qué transformaciones son necesarias efectuar en las mismas. Especialmente en un contexto en que éstas últimas sirven para expandir la difusión de las informaciones (Franquet y Villa Montoya, 2012b).

En un intento por aunar las perspectivas antes expuestas, Erdal (2012) aborda la producción informativa multiplataforma desde un cuádruple punto de vista que responde a la distinción entre un cross-media comunicativo (referido a los contenidos) y otro productivo (referido a estos procesos). Su propuesta contempla:

a. Un eje vertical, que abarca la producción de la información desde su origen hasta su difusión final y que tiene que ver con la polivalencia laboral del periodista.

b. Un eje horizontal, que se refiere a la difusión de la información a través de los diversos medios asociados al grupo y que tiene que ver con la distribución multiplataforma.

c. Una dimensión interna, que describe el proceso productivo de la información en el interior de la redacción multimedia y que tiene que ver con la organización y cooperación entre departamentos.

d. Una dimensión externa, que se orienta hacia los contenidos que consume el

ICONO14 | Año 2013 Volumen 11 N² 2 | ISSN: 1697-8293 | DOI: ri14.v1 1i2.582 
público para averiguar de qué manera se transforman en función del medio a través del cual se distribuyen.

En este punto, se pueden reutilizar las piezas elaboradas para un medio en otro sin cambios, combinarlas con otros materiales o reformularlas completamente (Boczkowski, 2004), ya sea de manera manual o automática. Sea cual sea la solución adoptada, la estrategia exige por parte de empresas y periodistas una cierta sensibilidad para aumentar el feedback, la transparencia y la colaboración con los usuarios (García Avilés, 2012). Unos usuarios que, en última instancia, son los que consumen estos contenidos y los distribuyen por otras plataformas, sociales, como Twitter: una fórmula cada vez más significativa en la manera que tienen de experimentar su participación en el proceso informativo (Hermida y otros, 2012).

\subsection{Twitter y web 2.0 en el Periodismo Institucional}

Twitter es una plataforma de microbloging que entró en funcionamiento en 2006. Permite que cualquier persona u organismo publique y difunda mensajes breves, de 140 caracteres como máximo, conocidos como tuits, entre sus seguidores, llamados followers. Java y otros (2007) listaron los usos más comunes de este servicio: chatear, conversar, compartir información y enviar noticias. Por su naturaleza dialógica, inherente al tratarse de una utilidad digital, Twitter se encuadra dentro de las herramientas de la denominada web 2.0.

Este término se acuñó a mediados de la primera década de este siglo para aludir al conjunto de aplicaciones web que se caracterizan por animar al usuario a participar y colaborar en la creación de contenido de manera que dejan de ser los consumidores pasivos que, hasta el momento y al menos teóricamente, eran. Ejemplos de esta filosofía son los blogs, las wikis o las redes sociales. Entre los principios clave que definen la web 2.0, 0'Reilly (2012), destaca su capacidad de controlar la inteligencia colectiva y de confiar en los usuarios a la hora de desarrollar servicios e informaciones. De hecho, son éstos últimos los que dan valor añadido a estas herramientas a través de su uso continuado.

Tradicionalmente, la comunicación dialógica ha servido de marco conceptual para estudiar el uso de las nuevas tecnologías en la comunicación institucional (Kent y Taylor, 2002; Seltzer y Mitrook, 2007; Park y Reber, 2008; Rybalko y Seltzer, 2010), ámbito al que se adscriben los gabinetes de prensa y comunicación.

DOI: ri14.v1 1i2.582 | ISSN: 1697-8293 | Año 2013 Volumen 11 N² 2 | ICONO14 
El motivo principal es que esta orientación permite explorar conceptos como el diálogo, la relación o la interacción entre los organismos y sus públicos a través de la web, los blogs 0 , más recientemente, los medios sociales. Y encaja perfectamente con otros postulados que defienden un ejercicio más honesto y ético de la comunicación por parte de las organizaciones gracias a un mayor contacto con sus públicos (Ledingham, 2003).

A grandes rasgos, Kent y Taylor definen la comunicación dialógica como un intercambio de ideas y opiniones, que se debe concretar efectivamente en el establecimiento de una serie de canales y mecanismos para que ésta tenga lugar (Kent y Taylor, 1998, 2002). Debido a su inherente naturaleza interactiva, el entorno digital se postula como el ideal para incentivar al diálogo. Estos autores, que a su vez son los principales impulsores de esta orientación, plantean cinco principios para conseguirlo a través de sitios web corporativos estratégicamente diseñados para ello (Kent y Taylor, 1998). Estos principios son:

1. Información útil y con valor añadido: las páginas web institucionales deben incluir datos que sean potencialmente valiosos para todos sus públicos, lo que en numerosas ocasiones se refleja en la manera en que los contenidos se estructuran y jerarquizan en el sitio. Este principio se asienta sobre la convicción que se visitan aquellos lugares que tienen algo que ofrecer a los usuarios: información útil, fiable y fácil de encontrar.

2. Generación de visitas de retorno: las webs deben contener información que anime a repetir su consulta como, por ejemplo, contenidos actualizados, foros, consultas en línea con expertos, etc. Se trata de poner en marcha estrategias interactivas, con formatos que exploten la posibilidad de pregunta-respuesta que ofrece el entorno digital.

3. Usabilidad: esta característica se refleja en la comodidad y facilidad de uso, pero también en unos contenidos fácilmente comprensibles, bien organizados y jerarquizados.

4. Crear un bucle dialógico: apela a la posibilidad de feedback inherente a la tecnología digital que permite a los usuarios preguntar a los organismos y a estos últimos contestar sus dudas y cuestiones. Para ello, deben procurar los recursos necesarios: formación adecuada a sus trabajadores para que respondan de manera profesional en el entorno virtual y una cierta implicación para efectivamente querer hacerlo.

ICONO14 | Año 2013 Volumen 11 N² 2 | ISSN: 1697-8293 | DOI: ri14.v1 1i2.582 
5. Conservación de visitas: este principio se relaciona con el tráfico que genera la página web, más concretamente, con evitar que los usuarios que entran en el sitio acaben por salir de él a través de los enlaces externos. Se aconseja señalar claramente el camino de vuelta y situar la publicidad en las partes periféricas de la página para evitar perder visitantes.

Por su carácter pionero, este modelo se ha empleado en las propuestas que estudian el uso de las nuevas tecnologías en la comunicación de las instituciones con sus públicos, incluyendo aquellas que se centran exclusivamente en los medios sociales. Un punto coincidente en todas ellas es la constatación de que los organismos suelen usar estas plataformas de manera unidireccional, con lo que el diálogo quedaría reducido a su mínima expresión. Así se ha detectado en el uso de Twitter por parte de las empresas que integran la lista Fortune 500 (Rybalko y Seltzer, 2010), de organizaciones sin ánimo de lucro (Waters y Jamal, 2011; Lovejoy y otros, 2012) o en instituciones de ámbito cultural como los museos (Capriotti y Kuklinski, 2012). Más recientemente, en su propuesta sobre la presencia de los colegios de médicos españoles en la web social, Sánchez-González y Paniagua-Rojano (2013) advertían que las organizaciones no están aprovechando aún de manera plena el potencial de estas herramientas. No debería de extrañarnos: Taylor y otros (2001) ya advirtieron hace una década de que los organismos con presencia virtual se volcaban más en aspectos técnicos y de diseño que en generar diálogo. Quizás, por falta de un plan estratégico de comunicación al respecto (Paniagua Rojano y Gómez Calderón, 2012).

En general, los organismos utilizan Twitter para enviar mensajes sobres sus actividades y seguir a periodistas (Briones y otros, 2011) y compartir información con los usuarios a través de mensajes que, de manera mayoritaria, los redirigen a la web corporativa (Waters y Jamal, 2011; Lovejoy y otros, 2012). Por ello y ante este panorama, Rybalko y Shelter (2010) aventuraron que quizás los principios enunciados por Kent y Taylor (1998) no sean útiles para analizar el diálogo de los organismos con sus públicos a través de las redes sociales.

En cualquier caso, las propuestas aquí expuestas no se detienen a examinar con detalle el circuito que los contenidos distribuidos por los organismos en cuestión. Ni tampoco describen las sinergias que se establecen con otros canales de comunicación externa como el teléfono, el correo electrónico o la página web, más allá de especificar el uso de enlaces y material multimedia en los tuits. No obstante,

DOI: ri14.v1 1i2.582 | ISSN: 1697-8293 | Año 2013 Volumen 11 N² 2 | ICONO14 
el marco genérico de la noción de cross-media permitiría explicar estas estrategias y desarrollarlas en contexto más amplio de la convergencia. Un fenómeno que también afecta a los gabinetes de prensa y comunicación como actores que son del proceso informativo. Las próximas líneas se centran en esta cuestión.

\section{Resultados}

El gabinete de prensa y comunicación del Servei Català de Trànsit (SCT) de la Generalitat de Catalunya nació a finales del año 2000. Sus principales funciones son informar sobre accidentes y retenciones en la red vial catalana así como organizar e implementar campañas y otras iniciativas (congresos, jornadas...) para concienciar a la población de la necesidad de una conducción segura. La oficina nació completamente digitalizada y desde el primer momento usó las utilidades tecnológicas en su quehacer informativo (página web, gestor de contenidos para la distribución de notas de prensa, fibra óptica para las imágenes...). Incorporó Twitter como herramienta de trabajo en diciembre de 2011 como parte de una estrategia de posicionamiento global en las redes sociales que contempla también el uso de Youtube (en marcha desde marzo de 2012) y la conversión de la revista en papel Infotrànsit al formato digital, sobre las mismas fechas. El organismo no tiene una única cuenta en la plataforma de microblogging sino una treintena, ya que se decidió abrir una por cada vía considerada como significativa (por ser de la red principal o soportar retenciones con frecuencia) y otra específica para el gabinete de prensa y comunicación (@transit).

El funcionamiento de todas ellas es diferente. Mientras que la de la oficina de prensa la mantienen las trabajadoras del departamento (todas mujeres), los tuits de las del resto se generan automáticamente. Esto es así porque el tratamiento de la información de tráfico referida a retenciones, cortes de carreteras u otras incidencias derivadas de las obras o inclemencias meteorológicas está altamente digitalizada. Existe una gran base de datos denominada Concentrador de Incidencias de Tráfico (CIT), gestionada por los trabajadores del Centro de Información Vial de Cataluña (CIVICAT), que permite publicar estos datos en la página web corporativa del organismo (www.gencat.cat/transit), almacenarnos para la confección de estadísticas sobre accidentalidad o cederlos a terceros para su explotación ${ }^{4}$. De aquí proceden los mensajes que se cuelgan en las cuentas pertenecientes a la red vial

ICONO14 | Año 2013 Volumen 11 N² 2 | ISSN: 1697-8293 | DOI: ri14.v1 1i2.582 
catalana. La información es, a grandes rasgos, similar a la que se obtiene consultando la página web sobre movilidad, ya que la información de base es la misma.

Por el contrario, del mantenimiento del perfil @transit se encarga únicamente y de manera manual (sin automatismos) el gabinete de prensa y comunicación. Esta cuenta tiene una triple función: informativa, preventiva y de difusión de las actividades informativas y publicitarias del gabinete (notas y ruedas de prensa, revista Infotrànsit y campañas de publicidad preventivas). A grandes rasgos, se han documentado tres usos:

a) La oficina difunde información sobre accidentes graves, mortales o excepcionales, que, en palabras de su principal responsable, "se salgan de lo normal". En estos casos, también se aprovecha para ampliar los datos sobre incidencias que se ofrecen en la web facilitando detalles como vehículos implicados o víctimas que allí no se recogen, con lo que se dota de valor añadido a los contenidos distribuidos a través de esta plataforma.

b) Asimismo, se distribuyen consejos sobre seguridad vial en aquellas situaciones que las circunstancias del tráfico así lo requieran como en caso de temporal de nieve, lluvia o de incendios forestales, por ejemplo. Esta cuenta también se hace eco de las campañas preventivas de las que al organismo le interesa que haya una "gran difusión viral", según su responsable de prensa y comunicación.

c) Finalmente, desde la cuenta se tuitean las notas y dosieres de prensa que se elaboran en el gabinete, los reportajes audiovisuales de la revista corporativa Infotrànsit, también disponibles a la página web corporativa del organismo.

En líneas generales, todos los tuits de los perfiles identificados como red vial (539 en total) distribuyen información sobre retenciones y se encuadran dentro de la categoría de "servicio". Cosa, por otra parte, lógica, ya que son los que se generan automáticamente a partir de la base de datos CIT y, por tanto, de contenido similar a lo que se recoge desde la web del organismo: es lo mismo aunque reelaborado. Aquí sólo aparecen informaciones sobre atascos, vías cortadas, obras, etc. No incluyen ningún enlace ni recurso audiovisual complementario.

De los 224 tuits de la cuenta del gabinete, todos ellos redactados por la oficina, la mayor parte (188, un 83,93\%) también se encuadrarían dentro de "servicio", ya que destacan alguna retención por su importancia, avisan de cortes de carril en caso de accidente, recuerdan rutas alternativas, etc. A estos habría que sumar los 8 mensajes $(3,57 \%)$ centrados únicamente en "sucesos", ya que informan sobre 
todo de la existencia de víctimas; y los 12 (5,36\%) que combinan ambas categorías. Apenas un $1 \%$ de los tuits (2) se destina a los "consejos" mientras que un $6,25 \%$ de ellos (14) se encuadrarían dentro de "prensa" al contener referencias a las actividades informativas del organismo (ruedas, notas, etc.) (ver Figura 1). Casi tres cuartas partes de estos últimos $(71,42 \%)$ contienen un enlace al material en sí colgado en la página web del organismo o en la sala de prensa corporativa del Gobierno de la Generalitat, del que depende el SCT. Con todo, sólo representa el $4,46 \%$ del total de mensajes de la cuenta @transit y el 1,31\% de todos los tuits analizados. Es el único recurso utilizado, ya que no se han documentado archivos audiovisuales (vídeos, fotografías o audios adjuntados en los tuits) en el resto de tuits encuadrados en "prensa".

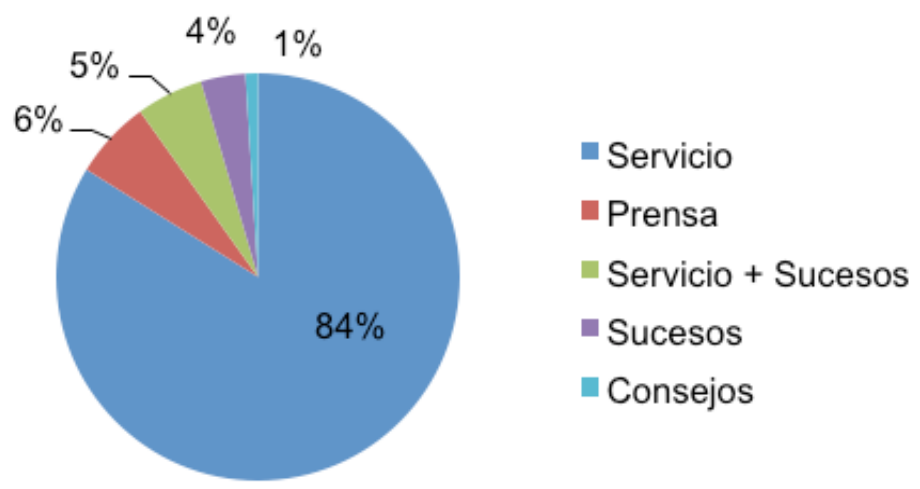

Figura 1: Tuits enviados por el gabinete (en porcentaje y por categorías).

Fuente: Elaboración propia

La estrategia más común en relación a los contenidos se identifica con la clonación, ya que se trata de la misma información distribuida a través de otra plataforma asociada al organismo de la misma manera que los medios asociados a un grupo clonan las piezas informativas. Todos los mensajes automáticos siguen esta lógica así como los encuadrados dentro de la categoría de "prensa", ya que estos últimos copian los titulares de las notas de prensa que se distribuyen por correo electrónico o a través de la sala de prensa corporativa. La inserción de enlaces en esta última categoría se puede explicar desde la promoción cruzada, ya que es una manera de invitar al usuario a consultar la web del organismo para conocer más detalles sobre lo que anuncia el tuit, necesariamente breve por las propias carac- 
terísticas técnicas del servicio.

No obstante, todos aquellos mensajes redactados por la oficina en relación a "servicio", "sucesos", "servicio y sucesos" y "consejos" son contenidos nuevos y diferentes, que no se corresponden a ninguna transformación de material previo. Como máximo, especialmente en casos de accidentes y retenciones graves, reinterpretan y amplían los datos consultables en el sitio. Esto es así porque no se reituitea todo, sino sólo aquello más significativo de lo allí publicado, con lo que, de alguna manera, jerarquizan los contenidos. Además, se aportan más detalles y se hace un seguimiento del suceso con constantes actualizaciones, cosa que no pasa en la página web: aquí el dato nuevo sustituye al anterior, no se acumula. En estos casos concretos, pues, estaríamos delante de una reformulación de materiales ya existentes.

Durante las dos semanas que duró el análisis del Twitter del SCT, no se detectó ninguna conversación pública con los usuarios, ya que, como se indicó desde la oficina, no se entra al diálogo público en estas plataformas a pesar de que la guía de usos y estilos de la Generalitat así lo aconseja (Generalitat, 2010). En Youtube, por ejemplo, la opción a comentar está deshabilitada. Con todo, casi la mitad de los mensajes $(41,96 \%)$ fueron retuiteados por los seguidores (194). Aunque las cuentas de Twitter de Trànsit no se dirigen a un colectivo específico, 43 de estos últimos $(22 \%)$ son seguidores o followers identificados en su perfil de cuenta como medios de comunicación (20), periodistas (10) u otros gabinetes de prensa (3). En los primeros cuatro meses de funcionamiento, el número de seguidores pasó de 1.943 a 5.391, según datos facilitados por el organismo durante el trabajo de campo ${ }^{5}$. Tanto los seguidores como los retuits serían, en este caso, indicadores que el gabinete tiene en cuenta para valorar el funcionamiento del servicio. Servirían a modo de indicador de feedback con la audiencia y, hasta incluso de legitimización del organismo.

\section{Discusión}

El uso de Twitter por parte del SCT se enmarca dentro de una estrategia general interna de producción y distribución de contenidos informativos marcada por la mecanización y el automatismo. Los materiales son accesibles a través de los diversos canales digitales externos del organismo (web, sala de prensa y redes

DOI: ri14.v1 1i2.582 | ISSN: 1697-8293 | Año 2013 Volumen 11 N² 2 | ICONO14 
sociales) a los que se une un recurso tradicional: la información facilitada telefónicamente. La elevada digitalización existente de los procesos de gestión de la información sobre movilidad existentes en el SCT hace posible que la mayor parte de los contenidos generados se trasvase automáticamente a las cuentas de Twitter. Y las transformaciones requeridas son mínimas, sólo evidentes cuando se trata del perfil específico gestionado por el gabinete de prensa. La oficina sólo debe estar atenta a aquellos accidentes o retenciones graves para informar de ellas y hacer un seguimiento específico a través de su perfil.

Llegado a este punto, recuperamos las hipótesis planteadas al inicio de esta propuesta para indicar que:

1. El uso de Twitter en el Periodismo Institucional descansa en una estrategia basada en reutilizar los contenidos disponibles en la web, sin apenas adaptaciones, para redistribuirlos a través de otra plataforma la mayoría de las veces redundante con respecto a otros canales de comunicación externa. Un dato que corrobora esto es que el $70,64 \%$ de los mensajes se generan de manera automática. Se trata de tener presencia y voz en un espacio nuevo aunque sin aprovechar todas las potencialidades del mismo, como por ejemplo la interactividad o el uso intensivo de enlaces o material audiovisual. Así, sólo un 4,46\% de los tuits de la oficina presenta algún recurso digital, siendo siempre éste un vínculo. El valor añadido informativo, cuando lo hay, se encuentra en aquellos contenidos configurados exclusivamente para el servicio de microblogging, que complementan los datos disponibles en la web (accidentes y retenciones graves).

2. Si bien las estrategias comunicativas implementadas por el gabinete a través de Twitter eluden la conversación en público con los usuarios, no renuncian a generar comunidad acumulando seguidores y generando retuiteos. No se ha documentado ninguna conversación pública y los únicos datos en relación a la interacción con la audiencia serían el número de seguidores (18.870 followers) los retuits (41,96\% de los mensajes de la cuenta gestionada íntegramente por el gabinete). Un uso paradójico para una plataforma basada en la participación y que tiene en el diálogo su filosofía fundacional de funcionamiento.

Podemos concluir que el SCT utiliza Twitter para informar sobre siniestralidad y las actividades informativas y preventivas desplegadas por el organismo en cumplimiento de sus funciones principales como institución orientada a la reducción de la siniestralidad y a garantizar la seguridad en la red vial catalana. Sus canales

ICONO14 | Año 2013 Volumen 11 N² 2 | ISSN: 1697-8293 | DOI: ri14.v1 1i2.582 
de comunicación externos se articulan alrededor de la página web corporativa, el principal motor que los impulsa. La plataforma de microblogging se perfila como una opción complementaria a la anterior, encargada de replicar los contenidos de la web en otro escenario. En este sentido, funciona más como un medio de información, con tuits publicados regularmente a modo de breves titulares, que como una red de comunicación entre individuos e instituciones.

\section{Notas}

[1] Este artículo forma parte de las actividades del proyecto de investigación P1-1B2010-53, financiado por la Fundació Caixa Castelló-Bancaixa y la Universitat Jaume I (Plan de Promoción de la Investigación, 2011-2013.

[2] El Servei Català de Trànsit (SCT) es un organismo autónomo adscrito al Departamento de Interior de la Generalitat de Catalunya cuyo principal cometido es controlar el tráfico y la seguridad vial en esta comunidad. Su gabinete de prensa nació el 1 de diciembre de 2000 completamente digitalizado desde el primer día de funcionamiento.

[3] También es habitual que el término cross-media aparezca ligado al de narrativas transmedia (Jenkins, 2008), un concepto aplicado especialmente a los contenidos vinculados al ocio (teleseries o películas). Ibrus y Scolari (2012) puntualizan al respecto que lo transmedia requiere la existencia de una única historia que se narra a través de varias plataformas que, a su vez, posibilitan la contribución del público.

[4] Esto es lo que pasa, por ejemplo, con Televisió de Catalunya, que elabora los recursos que utiliza para ilustrar sus crónicas sobre el estado del tráfico a partir de estos datos en bruto.

[5] En marzo de 2013, el número de seguidores asciende a 18.870 y de seguidos por el SCT a 7, con más de 6.500 tuits enviados, según consta en la propia cuenta [fecha de consulta: 9 de marzo de 2013].

\section{Referencias}

Boczkoswi, P. J. (2004). Digitizing the News. Innovation in Online Newspapers. Cambridge, MA: MIT Press.

Briones, R. L., Kuch, B., Fisher Liu, B. \& Jin, Y. (2011). Keeping up with the digital age: How the American Red Cross uses social media to build relationships. Public Relations Review, 37(1), 37-43. D0I: 10.1016/j. pubrev.2010.12.006.

DOI: ri14.v11i2.582 | ISSN: 1697-8293 | Año 2013 Volumen 11 N² 2 | ICONO14 
Capriotti, P.\& Kuklinski, H. (2012). Assessing dialogic communication through the Internet in Spanish museums. Public Relations Review, 38(4), 619-626. DOI: 10.1016/j.pubrev.2012.05.005.

Casero-Ripollés, A. (2010). Prensa en Internet: nuevos modelos de negocio en el escenario de la convergencia. El profesional de la información, 19(6), 595601. DOI: 10.3145/epi.2010.nov05.

--. (2012). Contenidos periodísticos y nuevos modelos de negocio: evaluación de servicios digitales. El profesional de la información, 21(4), 341- 346. D0I: 10.3145/epi.2012.jul.02.

Castro Monge, E. (2010). El estudio de casos como metodología de investigación y su importancia en la administración y dirección de empresas. Revista Nacional de Administración, 1(2), 31-54.

Chan-Olmsted, S. M.\& Chang, B.-H. (2003). Diversification Strategy of Global Media Conglomerates: Examining its Patterns and Determinants. Journal of Media Economics, 16(4), 213-233.

--. (2006). Mobile wireless strategy of media firms: examining the wireless diversification patterns of leading global media conglomerates. En: J. Groebel, E. M. Noam \& Feldmann, V. Mobile Media: Content and Services for Wireless Communications. Mahwah, NJ: Lawrence Erlbaum Associates, 165183.

Cottle, S. \& Ashton, M. (1999). From BBC newsroom to BBC newscenter: on changing technology and journalist practices. Convergence, 5 (3), 22-43. Doi: $10.1177 / 135485659900500304$.

Dailey, L., Demo, L. \& Spillman, M. (2005). The convergence continuum: A model for studying collaboration between media newsrooms. Atlantic Journal of Communication, 13(3), 150-168. DOI: 10.1207/s15456889ajc1303_2

Deuze, M. (2004). What is multimedia journalism? Journalism Studies, 5(2), 139152. DOI: $10.1080 / 1461670042000211131$.

Domingo, D. y otros (2007). Four Dimensions of Journalistic Convergence: A preliminary approach to current media trends at Spain. Comunicación presentada en el $8^{\circ}$ International Symposium on Online Journalism. Austin, 2007. 
Dupagne, M. \& Garrison, B. (2006). The meaning and influence of convergence. A qualitative case study of newsroom work at the Tampa News Center. Journalism Studies, 7(2), 237-255.

Erdal, I. J. (2008). Cross-media news journalism. 0slo: Universidad de 0slo. Tesis doctoral.

--. (2011). Coming to Terms with Convergence Journalism: Cross-Media as a Theoretical and Analytical Concept. Convergence, 17(2), 213-223. D0I: $10.1177 / 1354856510397109$.

--. (2012). Bridging the Gap: Toward a Typology of Cross-Media News Production Processes. En: Siapera, E. \& Veglis, A. The Handbook of Global Online Journalism. Malden, MA: Wiley-Blackwell, 179-191.

Franquet, R. \& Villa Montoya, M. I. (2012a). Interpretació i anàlisi de continguts cross-media : el cas de Televisió de Catalunya. Anàlisi, monográfico audiovisual 2.0, 49-63.

Franquet, R. \& Villa Montoya, M. I. (2012b). Exploring the crossmedia content of public broadcasters in Catalonia and Denmark. En: Ibrus, I. ; Scolari, C. A. Crossmedia innovations. Texts, Markets, Institutions. Frankfurt : Peter Lang, 277-296.

García Avilés, J. A. (2012). Innovation management in crossmedia production: Leading change in the newsroom. En: Ibrus, I. \& Scolari, C. A. Crossmedia innovations. Texts, Markets, Institutions. Frankfurt: Peter Lang, 259-276. García Avilés, J. A., León, B., Sanders K. \& Harrison, J. (2004). Journalists at Digital Television Newsrooms in Britain and Spain. Workflow and multiskilling in a competitive environment. Journalism Studies, 5(1), 87-100. D0I: $10.1080 / 1461670032000174765$.

--. (2009). Newsroom integration in Austria, Spain and Germany. Journalism Practice, 3(3), 285- 303. D0I: 10.1080/17512780902798638.

Garrison, B. (2003). How newspaper reporters use the web to gather news.

Newspaper Research Journal, 24 (3), 62-75.

Generalitat de Catalunya (2010). Guía de usos y estilo en las redes sociales de la Generalitat de Catalunya. Barcelona: Generalitat de Catalunya.

Hermida, A., Fletcher, F., Korell, D. \& Logan, D. (2012). Share, Like, Recommend. Decoding the social media news consumer. Journalism Studies, 13(5-6), 815824. DOI: 10.1080/1461670X.2012.664430. 
Huang, E., Rademakers, L.; Moshood A. F. \& Dunlap, L. (2004). Converged journalism and quality: A case study of the Tampa Tribune news stories. Convergence, 10(4), 73-91. D0I: 10.1177/135485650401000407

Huang, E., Davison, K., Shreve, S., Davis, T., Bettendorf, E. \& Nair, A. (2006). Facing the challenges of convergence: Media professionals' concerns of working across media platforms. Convergence, 12(1), 83-98. D0I: $10.1177 / 1354856506061557$

Ibrus, I. \& Scolari, C. A. (2012). Introduction : Crossmedia innovation? En: Ibrus, I. \& Scolari, C. A. Crossmedia innovations. Texts, Markets, Institutions. Frankfurt : Peter Lang, 7-21.

Infotendencias Group (2012). "Media convergence". En: Siapera, E.\& Veglis, A. The Handbook of Global Online Journalism. Malden, MA: Wiley-Blackwell, 2138.

Java, A., Finin, T., Song, X. \& Tseng, B. (2007). Why We Twitter: Understanding microblogging usage and communities. En: Proceedings of the 9th WebKDD and 1st SNAKDD 2007 workshop on Web mining and social network analysis (WebKDD/SNA-KDD '07), New York: ACM Press, pp. 56-65.

Jenkins, H. (2008). Convergence culture: la cultura de la convergencia de los medios de comunicación. Barcelona: Paidós.

Kent, M. L. \& Taylor, M. (1998). Building dialogic relationship through the World Wide Web. Public Relations Review, 24(3), 321-334.

--. (2002). Toward a dialogic theory of public relations. Public Relations Review, 28(1), 21-37. D0I: 10.1016/S0363-8111(02)00108-X.

Larrondo, A., Larrañaga-Zubizarreta, J., Meso, K. \& Agirreazkuenaga, I. (2012). Convergencia de medios y redacciones: el caso de la Radio Televisión Pública Vasca (EITB). El profesional de la información, 21(4), 347-353. D0I: 10.3145/ epi.2012.jul.03.

Ledingham, J. A. (2003). Explicating relationship management as a general theory of public relations. Journal of Public Relations Research, 15 (2), 181198. DOI: 10.1207/S1532754XJPRR1502_4.

Lovejoy, K., Waters, R. D. \& Saxton, G. D. (2012). Engaging stakeholders through Twitter: How nonprofit organizations are getting more out of 140 characters or less. Public Relations Review, 38 (2), 313-318. D0I: 10.1016/j. pubrev.2012.01.005.

ICONO14 | Año 2013 Volumen 11 N² 2 | ISSN: 1697-8293 | DOI: ri14.v1 1i2.582 
Marjoribanks, T. (2003). Strategizing technological innovation: The case of news corporation. En: Cottle, S. (ed.). Media Organization and Production. London: Sage, 59-75.

Marken, G. A. (1995). Getting the Most from Your Presence in Ciberspace, Public Relations Quarterly, 40(3), 36-37.

Merriam, S. B. (1988). Case Study Research in Education. San Francisco: Jossey Bass.

Micó, J. L., Masip, P. \& Domingo, D. (2013). To wish impossible things:

Convergence as a process of diffusion of innovations in an actornetwork. International Communication Gazette, 75(1), 118-137. D0I: $10.1177 / 1748048512461765$.

Moreno, A., Zerfass, A., Tench, R., Verčič, D. \& Verhoeven, P. (2009). European Communication Monitor. Current developments, issues and tendencies of the professional practice of public relations in Europe. Public Relations Review, 35 (1), 79-82. Doi:10.1016/j.pubrev.2008.12.001.

Moreno, A., Verhoeven, P., Tench, R. \& Zerfass, A. (2010). European

Communication Monitor 2009. An institutionalized view of how public relations and communication management professionals face the economic and media crises in Europe. Public Relations Review, 36 (2), 97-104. DOI:10.1016/j.pubrev.2010.02.004.

0'Reilly, T. (2012). What is Web 2.0? Design Patterns and Business Models for the Next Generation of Software. En: Mandiberg, M. The Social Media Reader. New York-London: New York University Press, 32-52.

Park, H. \& Reber, B. (2008). Relationship building and the use of Web sites: How Fortune 500 companies use their Web sites to build relations. Public Relations Review, 34(4), 409-411. D0I: 10.1016/j.pubrev.2008.06.006.

Paniagua Rojano, F. J. \& Gómez Calderón, B. J. (2012). Hacia la comunicación 2.0. El uso de las redes sociales por parte de las universidades españolas. Icono 14, 10(3), 346-364. D0I: 10.7195/ri14.v10i3.473

Rybalko, S. \& Seltzer, T. (2010). Dialogic communication in 140 characters or less: How Fortune 500 companies engage stakeholders using Twitter. Public Relations Review, 36(4), 336-341. D0I: 10.1016/j.pubrev.2010.08.004. 
Sánchez-González, M. \& Paniagua-Rojano, F.J. (2013). Estrategias de comunicación 2.0 en asociaciones profesionales. Estudio del caso de los Colegios Oficiales de Médicos en España. Revista Mediterránea de Comunicación, 4(1), 21-51. Recuperado el 10 de juno de 2013 de: http:// mediterranea-comunicacion.org/index.php?journal=Mediterranea\&page=articl e\&op=view\&path\%5B\%5D=40\&path\%5B \%5D=54

Seltzer, T \& Mitrook, M. A. (2007). The dialogic potential of weblogs in relationships building. Public Relations Review, 33(2), 227-229. D0I: 10.1016/j.pubrev.2007.02.011.

Singer, J. (2004). Strange bedfellows? The diffusion of convergence in four news organizations. Journalism Studies, 5(1), 3-18.

Taylor, M., Kent, M. L. \& White, W. J. (2001). How activist organizations are using the Internet to build relationships. Public Relations Review, 27(3), 263284. DOI: $10.1016 /$ S0363-8111(01)00086-8.

Verhoeven, P. (2012). How European PR practitioners handle digital and social media. Public Relations Review, 38 (1), 162-164. D0I:10.1016/j. pubrev.2011.08.015.

Wallace, S. (2013). The complexities of convergence: Multiskilled journalists working in BBC regional multimedia newsrooms. International Communication Gazette, 75 (1), 99-117. D0I:10.1177/1748048512461764.

Waters, R. D. \& Jamal, J. Y. (2011). Tweet, tweet, tweet: A content analysis of nonprofit organizations' Twitter updates. Public Relations Review, 37 (3), 321-324. D0I: 10.1016/j.pubrev.2011.03.002.

Yin, R. K. (1994). Case study research: Design and Methods. Thousand Oaks: Sage Publications.

Zerfass, A., Verčič, D., Verhoeven, P., Moreno, A. \& Tench, R. (2012). European Communication Monitor 2012. Challenges and Competencies for Strategic Communication. Results of an empirical survey in 42 countries. Bruselas: EACD. 